\title{
Aging effects on neural entrainment to a musical beat
}

Sauvé, S.A. ${ }^{a}$, Bolt, E.L.W. ${ }^{a}$, Nozaradan, S. ${ }^{b}$, Zendel, B.R. ${ }^{a, c}$

${ }^{a}$ Faculty of Medicine, Memorial University of Newfoundland, 300 Prince Philip Dr, St. John's, NL, A1B 3V6, Canada; sarah.sauve@mun.ca; ebolt@grenfell.mun.ca

${ }^{b}$ Institute of Neuroscience, Université Catholique de Louvain, Place de I'Université 1, 1348

Ottignies-Louvain-la-Neuve, Belgium ; sylvie.nozaradan@uclouvain.be

${ }^{\mathrm{c}}$ Aging Research Centre, Newfoundland and Labrador, Grenfell Campus, Memorial University, 20 University Dr, Corner Brook, NL A2H 5G5, Canada; bzendel@mun.ca

Corresponding author: S. A. Sauvé

Disclosure statement: The authors have no conflicts of interest to declare.

Funding: This work was supported by Dr. Zendel's Canada Research Chair in Aging and Auditory Neuroscience. 


\begin{abstract}
When listening to music, the brain entrains to the musical rhythm and produces neural activity at the beat frequency. Younger $(<35)$ and older $(>60)$ adults listened to slow $(1.25 \mathrm{~Hz})$ and fast $(2.5 \mathrm{~Hz})$ syncopated and non-syncopated rhythms while intermittently performing a tapping task. EEG was recorded and frequency tagging was employed to analyze meter-related and meter-unrelated frequencies elicited by the rhythms. The meter-related frequencies included the beat frequency (BF), its first three harmonics $(\mathrm{H} 1-\mathrm{H} 3)$ and the frequency of the whole pattern, or cycle rate $(\mathrm{CR})$ while the meter-unrelated frequencies included the remaining harmonics of the CR up to the eleventh harmonic. Age effects were observed at the BF, where younger adults had larger amplitudes than older adults and at the CR. At the fast tempo, older adults did not differentiate between the CR, the BF and H3. Together, these results suggest older adults experience a breakdown of selective encoding at the fast tempo and reliance on high-level information, exhibiting aspects of both the inhibition and compensation theories of aging.
\end{abstract}

Keywords: neural entrainment, aging, frequency tagging, EEG, rhythm perception 


\section{Introduction}

The ability to perceive musical beat is necessary to make predictions about upcoming events in music and is an integral part of music perception (Huron, 2006). When listening to music, listeners entrain to regularities that are organized into a meter. A metrical structure consists of a pattern of strong and weak beats, which can be, but are not necessarily, present in the acoustic signal itself (Large, 2008). Increasing evidence suggest that meter and beat perception may be related to selective enhancement of neural activity at the meter and beat frequencies and their harmonics, as observed by frequency tagging the EEG signal recorded while listeners are presented with a rhythmic pattern inducing perception of a given metrical structure (Lenc, Keller, Varlet \& Nozaradan, 2018; Nozaradan, Peretz, Missal, \& Mouraux, 2011; Nozaradan, Peretz \& Moureaux, 2012; Nozaradan, Peretz \& Keller, 2016). Referred to as neural entrainment, his selective neural activity at meter- and beat-related frequencies has been observed not only in regular rhythms with high acoustic energy at the beat frequency but also in syncopated rhythms, where silence could occur at the expected beat, suggesting that this selective enhancement at beat-related frequencies cannot be fully explained by acoustic features alone (Nozaradan, Keller, Rossion \& Mouraux, 2018; see also Lenc, Keller, Varlet, \& Nozaradan, 2018; Nozaradan, Peretz, \& Keller, 2016). In this paper, we refer to neural entrainment as frequency coupling between input (acoustic information) and output (neural activity); we do not here seek to infer any particular generating mechanism underlying the phenomenon.

Little is known about neural entrainment in older adults. Aging has a generally negative effect on cognitive skills such as working memory capacity (Gazzaley, Cooney, Rissman, \& D’esposito, 2005; Hasher \& Zacks, 1988; Salthouse, 1990, 1994; Salthouse \& Babcock, 1991) and processing speed (Finkel, Reynolds, McArdle, \& Pedersen, 2007; Salthouse, 1996), while crystalized knowledge increases (Craik \& Salthouse, 2011). On the other hand, the little evidence we have investigating music perception in older adults suggests that it is generally preserved (Halpern, Bartlett, \& Dowling, 1995, 1998; Halpern, Kwak, 
Bartlett, \& Dowling, 1996; Halpern et al., 2017). In some cases the performance of a musical task is preserved, but the underlying brain activity changes, suggesting that older adults may automatically compensate for declining cognitive or perceptual abilities in the musical domain (Halpern et al., 2017; Lagrois, Peretz, \& Zendel, 2018). This may be due to the development of compensatory mechanisms similar to those found for speech in noise perception, where older adults use contextual information to help overcome age-related changes in hearing and cognitive abilities (Birren \& Fisher, 1995; Horn, 1982; Horn \& Cattell, 1967; Madden, 1992; Pichora-Fuller, Schneider, \& Daneman, 1995). When rating the fit of a note in a melody, older adults rely more heavily on scale degree information, derived from innate knowledge of the tonal hierarchy, whereas younger adults rely more heavily on note-to-note information (Bharucha \& Krumhansl, 1983; Halpern et al., 2017). In addition to increased use of contextual and crystalized knowledge, the neural compensation theory of aging stems from the observation of patterns of over- and under-activation, reduced asymmetry and reduced connectivity of brain activity in the brains of older adults as compared to younger adults performing the same task (Cabeza, 2002; Cabeza et al., 2004; Davis, Kragel, Madden, \& Cabeza, 2011; Grady, Mclntosh, \& Craik, 2003; Reuter-Lorenz, 2002; Reuter-Lorenz \& Cappell, 2008; Reuter-Lorenz et al., 2000).

In terms of rhythm perception, Sauvé et al. (2019) used frequency-tagging to compare neural activity in older and younger adults while passively listening to a metronome. This study found that older adults, compared to younger adults, had reduced amplitude of neural activity at the frequency of the metronome (i.e., beat frequency) and a smaller drop off in amplitude from the beat frequency to the first three harmonics of the beat frequency (Sauvé et al., 2019). Critically, these differences were not due to age-related changes in hearing as measured by pure-tone thresholds. These results were interpreted as support for the inhibition theory of aging, where aging reduces the neural inhibition of harmonics that arise due to the natural behaviour of harmonic oscillators (Hasher, 2015; Salthouse \& Meinz, 1995). More generally, the inhibition theory of aging refers to a decline in the ability to ignore 
irrelevant information in the context of a task, a decline in the ability to control one's locus of attention and an inability to effectively suppress inappropriate responses (e.g. in the Stroop task; Lustig, Hasher, \& Zacks, 2007). In this case, the larger amplitude of neural activity at the beat's harmonics and corresponding lack of selectivity of the beat frequency in older adults as compared to younger adults seems to be evidence of a lack of ability to automatically enhance the most relevant information (i.e. the beat) (Sauvé et al., 2019).

The goal of the current study was to explore differences in neural entrainment between older adults and younger adults actively listening to a rhythm that contains acoustic energy at the beat (i.e. non-syncopated) and when there is less acoustic energy at the beat frequency, and the perception of beat is derived endogenously (i.e., syncopated; see Lenc et al. 2018). Based on our previous findings (Sauvé et al., 2019), we expect to see smaller amplitudes for neural activity at the beat frequency, or larger amplitudes for neural activity at the harmonics of the beat frequency, in older adults compared to younger adults. Additionally, given older adults' increased knowledge about musical patterns, it is hypothesized that older adults will have smaller differences between neural amplitudes at the beat frequency for endogenously and exogenously derived beats (i.e., syncopated vs. non-syncopated) compared to younger adults.

\section{Materials and methods}

\subsection{Participants}

Twenty-nine participants (same as Sauvé et al., 2019) participated in this study and provided written informed consent in accordance with the Interdisciplinary Committee on Ethics in Human Research at Memorial University of Newfoundland. Participants were divided into two age groups: 15 older adults (>60 yrs; 10 female) and 14 younger adults (<25 yrs; 7 female). All participants self-reported being healthy, right-handed and free of any cognitive deficit. All participants were non-musicians although 
some had music training in childhood. Hearing abilities were assessed using pure-tone (PT) audiometry. All participants received a small cash honorarium for their participation. Table 1 summarizes participant demographics.

Table 1. Participant Demographics

\begin{tabular}{|c|c|c|c|}
\hline & Age & Formal Education (Years)* & Pure-tone Average (dB HL)** \\
\hline Younger Adults & $20.3(1.84)$ & $14.6(2.23)$ & $6.2(5.07)$ \\
\hline Older Adults & $63.4(3.68)$ & $12.87(3.46)$ & $13.28(9.03)$ \\
\hline \multicolumn{4}{|c|}{${ }^{*} \mathrm{t}(28)=1.6, \mathrm{p}=.12 ;$ standard deviation in brackets } \\
\hline \multicolumn{4}{|c|}{$* *$ Binaural average of pure-tone threshold at $500,1000 \& 2000 ; \mathrm{t}(28)=40.55, \mathrm{p}<.001$; standard } \\
\hline
\end{tabular}

\title{
2.2 Stimuli
}

\author{
Non-syncopated $\mathrm{X} . \mathrm{X} X \mathrm{X} . \mathrm{X} X \mathrm{X} . \mathrm{X}$ \\ Syncopated $\mathrm{XXXX} . \mathrm{XXX} . . \mathrm{X}$.
}

Figure 1. Non-syncopated and syncopated rhythms containing three groups of four events where an $X$ represents a sound and a . represents silence.

Stimuli were similar to that of Nozaradan et al.'s (2016) and divided into four conditions: two rhythms (non-syncopated and syncopated) at two tempi (slow; $1.25 \mathrm{~Hz}$ and fast; $2.5 \mathrm{~Hz}$ ). Nozaradan et al (2016) also included a slower condition $(0.6 \mathrm{~Hz})$, and a faster condition $(3.8 \mathrm{~Hz})$. We excluded these conditions because there was no impact of tempo on neural activity during non-syncopated rhythms, and the impact of tempo on neural activity for syncopated rhythms was linear, and could thus be observed with two conditions. Moreover, because we were testing older adults we wanted to minimize testing time as much as possible. The structure of the rhythms was based on the alternation of 12 
events, consisting of sounds and silent intervals. The sounds consisted of $1000 \mathrm{~Hz}$ pure tones, presented at $\sim 75 \mathrm{~dB}$ SPL, with $10 \mathrm{~ms}$ rise and fall times and were either $100 \mathrm{~ms}$ or $200 \mathrm{~ms}$ long for the fast or slow tempo, respectively. All stimuli were presented via insert earphones (Etymotic E3A). As described in Nozaradan et al. (2016), rhythms were designed to induce the perception of a beat based on a preference for grouping by 4 events with 3 such beats per cycle. The first rhythm was non-syncopated, with regular structure congruent with the beat periodicity, while the second rhythm was syncopated, with no prominent acoustic structure matching the beat periodicity. At the slow tempo, each cycle was $2400 \mathrm{~ms}$, with a beat rate of $800 \mathrm{~ms} /$ beat $(1.25 \mathrm{~Hz})$, and at the fast tempo each cycle was $1200 \mathrm{~ms}$ with a beat rate of $400 \mathrm{~ms} /$ beat $(2.5 \mathrm{~Hz})$. Stimuli were generated in Reaper ( $V$ 5.16), and presented to participants using Eprime (V 3.0).

\subsection{Procedure}

After providing written informed consent, participants completed a short demographics questionnaire, and were given the possibility to practice tapping to the 4 rhythms that would be presented during the study. Each participant was then fitted with a 128-channel BioSemi EEG cap and 6 external electrodes located at inferior ocular (IO1, IO2), lateral ocular (LO1, LO2) and mastoid (M1, M2) positions. Next the participant was seated in a double-walled, electrically shielded sound-attenuating booth (Eckel). Participants were given a response box (Chronos, EPrime), and could see a computer monitor. Participants were told to attend to the rhythm, and to try and maintain the beat mentally so they could tap a finger along to the beat. They were further instructed to tap their finger on one of the response buttons whenever the green light came up on the response box, and the computer monitor said, "Tap". When the response box buttons became red, and the computer monitor said "Stop Tapping", participants were instructed to stop tapping, but to maintain the beat mentally. To avoid fatigue associated with maintaining fixation for long periods of time, participants were told to either fixate on the monitor or response box during the task, and to minimize switching between the two. Each 
participant was told that they would be expected to start tapping as soon as the green light came back on. The two rhythms (non-syncopated, syncopated) were presented at the two tempi (slow, fast) in four separate blocks. The order of the blocks was identical for each participant (non-syncopated rhythm, tempo $1.25 \mathrm{~Hz}$ and $2.5 \mathrm{~Hz}$, and then syncopated rhythm, tempo $1.25 \mathrm{~Hz}$ and $2.5 \mathrm{~Hz}$ ). A fixed order was used to ensure participants could tap to the stimuli during the tapping phase. During pilot testing we found that participants had increased difficulty tapping at higher tempos, and to the syncopated compared to the non-syncopated rhythm. We found that a short practice session was not sufficient for participants tap accurately. Further pilot testing revealed that by slowly increasing the difficulty during the data collection, participants were able to tap comfortably. Ensuring that participants could tap to the beat appropriately helps ensure that the participants could accurately perceive the beat. When examining the neural activity, ensuring that both groups of participants could perceive the same beat was critical. At the start of each block, the participant was asked to tap a button lit in green on a response box to the beat of the rhythm for 48 seconds. After 48 seconds the buttons on the box became red, and a computer screen said "Stop Tapping". This 'rest' phase lasted 48 seconds, until the next tapping phase started, which lasted 12 seconds. There were a total of 10 resting phases, each followed by a tapping phase. This tapping task accompanied the listening task to ensure task engagement, where participants were instructed to tap with their dominant hand where they felt a natural beat.

\subsection{EEG data collection}

Neuroelectric brain activity was digitized at a sampling rate of $1024 \mathrm{~Hz}$ from 134 electrodes using the radial layout system, with a highpass filter set at $0.1 \mathrm{~Hz}$ using a Biosemi ActiveTwo system (Biosemi Inc., Amsterdam, Netherlands). Six electrodes were placed bilaterally at mastoid, inferior ocular and lateral ocular sites. 
Data was analysed using the Letswave 6 (Mouraux \& lannetti, 2008) signal processing toolbox for Matlab. First, EEG data was highpass filtered at $0.1 \mathrm{~Hz}$. Next, eye blinks and eye movements were identified for each participant using an independent component analysis (ICA). Components related to eye movements were removed from the EEG signal. Next the EEG data were referenced to the average. Each condition was divided into ten 48 second blocks (the resting phases), and then averaged into a single 48 second epoch, yielding 4 epochs (non-syncopated slow, syncopated slow, non-syncopated fast, syncopated fast) per channel and participant. EEG data recording during the tapping phase was excluded from analysis, as the purpose of the tapping task was to ensure the participant was attending to, and imagining a beat during the resting phase. To calculate the frequency-tagged response, a fast Fourier transform (FFT) was applied to all four epochs with a resulting spectral resolution of approximately $0.02 \mathrm{~Hz}$. These were then baseline corrected using a signal-to-noise ratio baseline analysis. This function expresses the amplitude of a frequency spectra relative to the amplitude of the spectra obtained from adjacent frequency bins (Mouraux et al., 2011). The amplitude of neural activity obtained from this analysis will be referred to as simply amplitude throughout this paper.

For each condition the peak spectral amplitudes at targeted frequency bins were extracted from a montage of frontocentral Electrodes (A1 [Cz], C1, C2, C11, C12, C21 [Fz], C22, C23, 24, C25, D1, D2) selected based on signal distribution (see Figure 3) and consistent with previous work (Lenc et al., 2018; Nozaradan et al., 2016, 2012). As in Lenc et al. (2018), 12 distinct peaks were extracted, corresponding to the repetition frequency of the whole pattern $(0.416 \mathrm{~Hz} ; 0.833 \mathrm{~Hz})$ and its harmonics up to the frequency of repetitions of single events $(5 \mathrm{~Hz} ; 10 \mathrm{~Hz})$. These were divided into meter-related frequencies and meter-unrelated frequencies. Meter-related frequencies include the beat frequency (BF; $1.25 \mathrm{~Hz} ; 2.5 \mathrm{~Hz}$ ), its first three harmonics (H1-H3; slow: $2.5 \mathrm{~Hz}, 3.75 \mathrm{~Hz}, 5 \mathrm{~Hz}$; Fast $5 \mathrm{~Hz}, 7.5 \mathrm{~Hz}, 10$ $\mathrm{Hz}$ ) and the frequency of the measure, or whole pattern (cycle rate: $\mathrm{CR} ; 0.416 \mathrm{~Hz} ; 0.833 \mathrm{~Hz}$ ). The remaining frequencies are meter-unrelated. Analysis bins were $0.1 \mathrm{~Hz}$ wide, corresponding to each 
target frequency peak plus or minus $0.05 \mathrm{~Hz}$. These bin widths were selected based on visual inspection of the data, where exact peak location varied within $0.1 \mathrm{~Hz}$ of the target frequency across participants.

\subsection{Data analysis}

EEG data was analyzed using mixed effects multiple linear regression modelling implemented in the R (3.3.2) Ime4 (Bates, Mächler, Bolker, \& Walker, 2015) package. Each model was maximally fitted to reflect the experimental design, as per Barr et al. (2013), where all models included random intercepts on participants and random slopes on participants by stimulus condition. In cases where the model did not converge or had a large eigenvalue ratio and a model without random slopes is no worse than the fully specified model, the model including random slopes is reported (Sauvé, Sayed, Dean, \& Pearce, 2018). All categorical variables were factors, where each level was compared to a base level. These base levels are slow for tempo, non-syncopated for rhythm, younger for age group, meter-related for frequency type and BF for peak. Models were evaluated using Pearson's correlation between the model's predictions and the data along with the correlation's 95\% Cls. Variance explained by each model was tested by calculating the coefficient of determination $R^{2}$. Statistical significance of each model was tested by a likelihood-ratio test between a null model (intercept and random effects) and the maximally fitted model. Statistical significance of each individual factor level for a given predictor was evaluated using $95 \% \mathrm{Cls}$, where an interval not including zero indicates a significant predictor. In each analysis, follow-up independent sample two-tailed t-tests with Bonferroni correction were conducted to investigate simple effects of age.

\subsubsection{Frequency response analysis.}

We first compare the amplitude of the response at meter-related and meter-unrelated frequencies to demonstrate selective enhancement of brain activity at meter-related frequencies for all stimuli, regardless of the physical structure of the stimulus, which in the case of the syncopated rhythm, 
does not include prominent meter-related frequencies. To do so, a model predicting spectral amplitude of neural activity included frequency type (meter-related, meter-unrelated), tempo (slow, fast), rhythm (non-syncopated, syncopated) and age group (younger, older) with fixed effects including interactions and PTAv (pure tone threshold average) score and channel as fixed main effects. PTAv is calculated by taking the binaural average of pure-tone threshold at 500, $1000 \& 2000 \mathrm{~Hz}$ and is included to check for low-level effects of poorer hearing in older adults while channel accounts for variance between electrodes.

Next, to investigate age effects on meter-related entrainment, a model predicting spectral amplitude of neural activity in response to meter-related frequencies included tempo (slow, fast), rhythm (non-syncopated, syncopated), peak (CR, BF, H1, H2, H3), age group (younger, older), PTAv score and channel as fixed effect. In all models, interactions with PTAv and channel were not modelled.

\subsubsection{Tapping analysis.}

The tapping task was included to ensure participants attended to the rhythmic sequences and maintained a beat perception. Only a small number of taps were recorded between each rest period, and a proper analysis of tapping behaviour requires significantly more taps (i.e. $>100$ in Zendel, Ross \& Fujioka, 2011). The tapping data may therefore be unreliable, and are presented as supplementary materials (see Supplementary Materials 2).

\section{Results}

\subsection{Meter-related vs. Meter-unrelated frequencies.}

The model reported in Table 2 predicts spectral amplitude of neural activity extracted at meterrelated and meter-unrelated frequencies for all participants in all conditions. The model has $r=.44, \mathrm{Cl}$ $[.43, .45]$ and $\mathrm{R}^{2}=.19$ and is significantly better than a null model, $\chi^{2}(27)=2423.1, \mathrm{p}<.001$. For brevity, 
only significant predictors with $\mathrm{R}^{2}$ above .01 are included in Table 2; full tables can be found in Supplementary Materials 1. Main effects of tempo (slow > fast), rhythm (nonsyncopated > syncopated) and frequency type (meter-related > meter-unrelated) were significant while age group, PTAv and channel were not. Significant interactions include two-way interactions between rhythm and frequency type and tempo and rhythm while a three-way interaction between tempo, rhythm and frequency type was also significant. There was no main effect of age, nor any significant interaction involving age. Figure 2 illustrates the mean amplitude of neural activity for all meter-related and meter-unrelated frequencies at slow and fast tempi for older and younger participants.

Table 2. Summary of significant predictors with $R^{2}>.01$ from the maximally fitted multiple linear regression model predicting spectral amplitude for meter-related and meter-unrelated peak frequencies including coefficient, Wald $95 \%$ confidence intervals and $\mathrm{R}^{2}$ for each predictor (where there are multiple levels, $R^{2}$ is given for the predictor as a whole). Random effects account for $<0.00$ variance and are therefore not reported.

\begin{tabular}{lcccc}
\multicolumn{1}{c}{ Predictor } & Coefficient & $\mathbf{2 . 5 \%}$ & $\mathbf{9 8 . 5 \%}$ & $\mathbf{R}^{\mathbf{2}}$ \\
\hline Intercept & 0.12 & 0.10 & 0.13 & .08 \\
Frequency Type & -0.06 & -0.07 & -0.06 & .09 \\
FType:Tempo & 0.004 & -0.004 & 0.01 & .01 \\
\hline
\end{tabular}

\subsection{Meter-related Frequencies.}

The model reported in Table 4 predicts spectral amplitude of neural activity extracted at meterrelated peak frequencies for all participants in all conditions. The model has $r=.61, \mathrm{Cl}[.60, .63]$ and $\mathrm{R} 2=$ .38 and is significantly better than a null model, $\chi 2(51)=2368.9, p<.001$. For brevity, only significant predictors with R2 above .01 are included in Table 4; full tables can be found in Supplementary Materials 1 along with corresponding summary statistics. Significant predictors include main effects of rhythm (nonsyncopated $>$ syncopated), age group (younger $>$ older) and frequency peak $(\mathrm{CR}=\mathrm{BF}>\mathrm{H} 3>$ $\mathrm{H} 1=\mathrm{H} 2$ ) as well as multiple two-, three- and four-way interactions. To further understand the interactions of age group with tempo, rhythm and frequency peak, follow-up t-tests were conducted (see Table 5). Age effects were observed at the BF in all tempo and rhythm conditions, where younger 

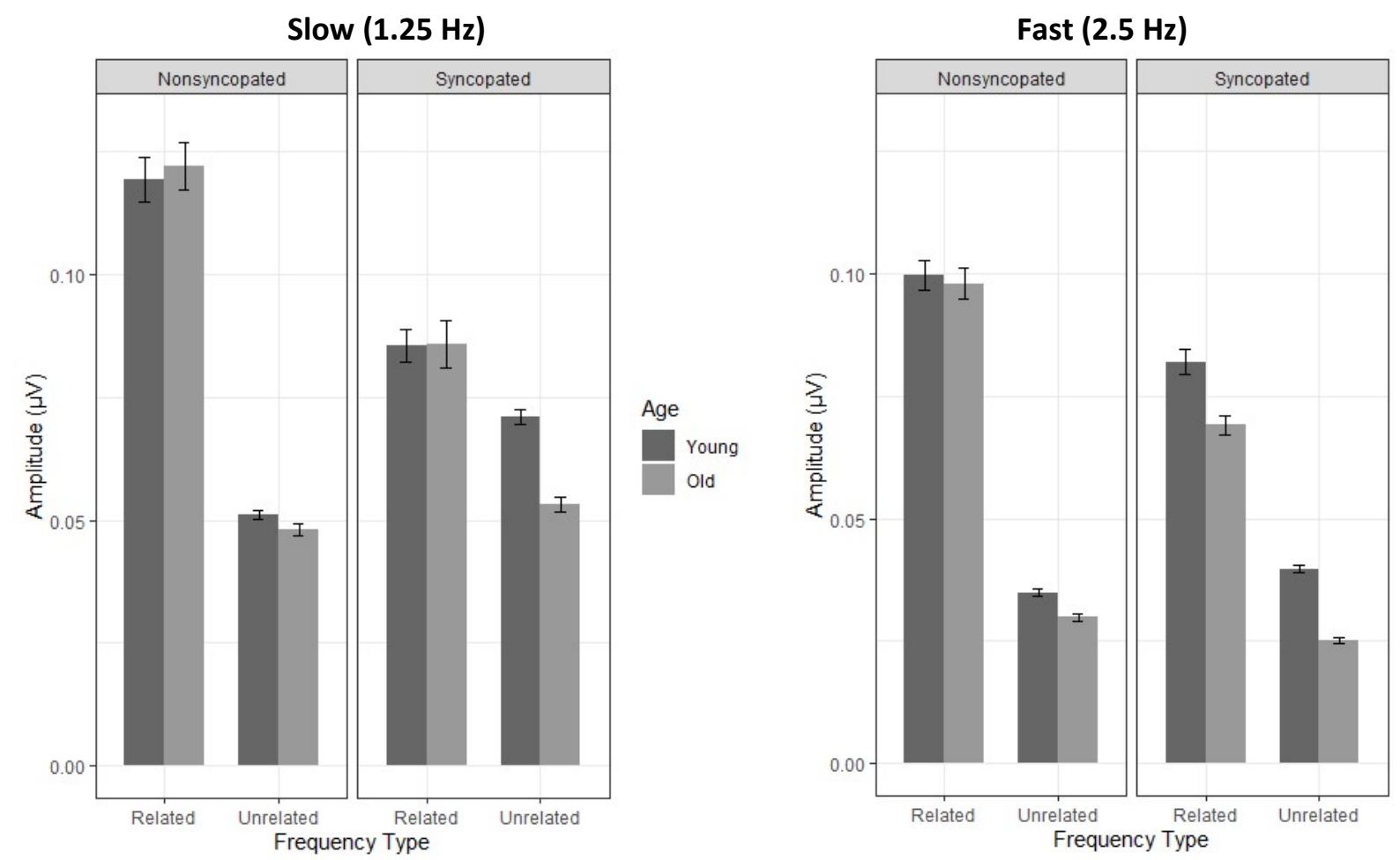

Figure 2. Mean amplitude of neural activity for all meter-related and meter-unrelated frequencies for slow and fast tempi and older and younger participants. Error bars indicate standard error of the mean.

adults had larger neural activity amplitudes than older adults. Younger adults also had significantly larger neural activity amplitudes than older adults at $\mathrm{H} 1$ in all conditions except fast non-syncopated. The opposite age effect was observed at $\mathrm{H} 3$ in all conditions except slow syncopated, and at CR in all conditions except fast syncopated, where here older adults had larger neural activity amplitudes than younger adults. In summary, overall, younger adults had larger neural activity amplitudes than older adults at the $\mathrm{BF}$ and $\mathrm{H} 1$ while older adults had larger neural activity amplitudes than younger adults at the CR and H3. Figure 3 illustrates mean neural activity amplitude for younger and older adults across tempo, rhythm and frequency peak, where significant age effects are annotated with asterisks while Figures 4 and 5 illustrate the brain response along with topographic maps for each meter-related frequency peak for the slow and fast tempi respectively. 
Table 4. Summary of significant predictors with $\mathrm{R}^{2}>.01$ from the maximally fitted multiple linear regression model predicting neural activity amplitude at meter-related frequencies, including coefficient, Wald $95 \%$ confidence intervals and $R^{2}$ for each predictor (where there are multiple levels, $R^{2}$ is given for the predictor as a whole). Random effects account for $<0.00$ variance and are therefore not reported.

\begin{tabular}{lcccc}
\multicolumn{1}{c}{ Predictor } & Coefficient & $\mathbf{2 . 5 \%}$ & $\mathbf{9 8 . 5 \%}$ & $\mathbf{R}^{\mathbf{2}}$ \\
\hline Intercept & 0.21 & 0.18 & 0.24 & .13 \\
CR & -0.04 & -0.06 & -0.02 & \\
H1 & -0.14 & -0.16 & -0.13 & .19 \\
H2 & -0.16 & -0.18 & -0.14 & \\
H3 & -0.12 & -0.14 & -0.10 & \\
Age: $\mathrm{CR}$ & 0.13 & 0.10 & 0.16 & .01 \\
Age: $\mathrm{H} 1$ & 0.05 & 0.02 & 0.07 & \\
Age:H2 & 0.07 & 0.04 & 0.09 & \\
Age:H3 & 0.10 & 0.07 & 0.12 & .02 \\
Tempo:CR & -0.03 & -0.06 & -0.009 & \\
Tempo:H1 & -0.002 & -0.02 & 0.02 & \\
Tempo:H2 & 0.01 & -0.01 & 0.04 & \\
Tempo:H3 & 0.01 & -0.008 & 0.04 & \\
\hline
\end{tabular}

Table 5. Follow-up t-test results for neural activity amplitudes at meter-related frequencies comparing age groups at each peak and condition including t-statistic, p-value and Cohen's d effect size.

\begin{tabular}{ccccc} 
Peak & Condition & t-statistic & p-value & Cohen's d \\
\cline { 2 - 5 } CR & Slow - Nonsyncopated & -2.83 & 0.004 & -0.29 \\
BF & Slow - Nonsyncopated & 5.41 & $<.001$ & 0.56 \\
H1 & Slow - Nonsyncopated & 3.22 & 0.001 & 0.35 \\
H2 & Slow - Nonsyncopated & -1.14 & 0.25 & -0.11 \\
H3 & Slow - Nonsyncopated & -4.45 & $<.001$ & -0.48 \\
\hline CR & Fast - Nonsyncopated & -0.64 & .52 & -0.06 \\
BF & Fast - Nonsyncopated & 4.74 & $<.001$ & 0.49 \\
H1 & Fast - Nonsyncopated & -0.03 & .96 & -0.004 \\
H2 & Fast - Nonsyncopated & 0.64 & .52 & 0.06 \\
H3 & Fast - Nonsyncopated & -3.63 & $<.001$ & -0.39 \\
\hline CR & Slow - Syncopated & -1.99 & 0.04 & -0.20 \\
BF & Slow - Syncopated & 3.48 & $<.001$ & 0.37 \\
H1 & Slow - Syncopated & 4.98 & $<.001$ & 0.52
\end{tabular}




\begin{tabular}{ccccc} 
H2 & Slow - Syncopated & 2.75 & .006 & 0.29 \\
H3 & Slow - Syncopated & -0.08 & 0.93 & -0.009 \\
\hline CR & Fast - Syncopated & 4.61 & $<.001$ & 0.47 \\
BF & Fast - Syncopated & 7.24 & $<.001$ & 0.74 \\
H1 & Fast - Syncopated & 7.10 & $<.001$ & 0.75 \\
H2 & Fast - Syncopated & -0.39 & .69 & -0.04 \\
H3 & Fast - Syncopated & -5.06 & $<.001$ & -0.52 \\
\hline
\end{tabular}

Finally, in order to further characterize the amplitude profiles, t-tests with Bonferroni correction were conducted between $\mathrm{BF}$ and $\mathrm{CR}$ and $\mathrm{BF}$ and $\mathrm{H} 3$ amplitudes for each age group and condition (see Tables 6 and 7). Younger adults have larger neural activity amplitudes at the BF than at the CR (except in the slow syncopated condition) while older adults present the opposite pattern in the slow conditions. When comparing BF and $\mathrm{H} 3$ peaks, there was always a significant difference in neural activity amplitude in younger adults while in older adults the only significant difference in these peaks was in the fast nonsyncopated condition, demonstrating an overall lack of differentiation between the BF and its third harmonic in older adults. Furthermore, there is a lack of differentiation between the CR, BF and $\mathrm{H} 3$ in older adults in the fast conditions but not the slow conditions; in the latter, the $C R$, or frequency of the entire pattern or measure, has a higher amplitude of neural activity than all other frequencies. This pattern of results demonstrates an overall lack of differentiation between beat and meter frequencies in older adults, where such differentiation is clear in younger adults.

Table 6. Follow-up t-test results for meter-related peak frequencies comparing BF and CR for each age group and condition including t-statistic, $p$-value and Cohen's d effect size.

\begin{tabular}{ccccc} 
Age Group & Condition & t-statistic & p-value & Cohen's d \\
\hline \multirow{2}{*}{ Young } & Slow - Non-syncopated & -2.42 & .01 & -0.26 \\
& Fast - Non-syncopated & -7.52 & $<.001$ & -0.74
\end{tabular}




\begin{tabular}{|c|c|c|c|c|}
\hline & Slow - Syncopated & 4.44 & $<.001$ & 0.47 \\
\hline & Fast - Syncopated & -6.53 & $<.001$ & -0.69 \\
\hline \multirow{4}{*}{ Old } & Slow - Non-syncopated & 4.44 & $<.001$ & 0.46 \\
\hline & Fast - Non-syncopated & -2.20 & .02 & -0.23 \\
\hline & Slow - Syncopated & 5.88 & $<.001$ & 0.59 \\
\hline & Fast-Syncopated & -3.05 & .002 & -0.31 \\
\hline
\end{tabular}

Table 7. Follow-up t-test results for meter-related peak frequencies comparing BF and $\mathrm{H} 3$ for each age group and condition including t-statistic, p-value and Cohen's d effect size.

\begin{tabular}{ccccc} 
Age Group & Condition & t-statistic & p-value & Cohen's d \\
\hline \multirow{3}{*}{ Young } & Slow - Non-syncopated & 10.36 & $<.001$ & 1.01 \\
& Fast - Non-syncopated & 11.14 & $<.001$ & 1.01 \\
& Slow - Syncopated & 3.30 & .001 & 0.35 \\
& Fast - Syncopated & 9.59 & $<.001$ & 0.95 \\
\hline \multirow{2}{*}{ Old } & Slow - Non-syncopated & 2.33 & .02 & 0.23 \\
& Fast - Non-syncopated & 3.24 & .001 & 0.36 \\
& Slow - Syncopated & 0.91 & .36 & 0.09 \\
& Fast - Syncopated & -1.95 & .05 & -0.19 \\
\hline
\end{tabular}



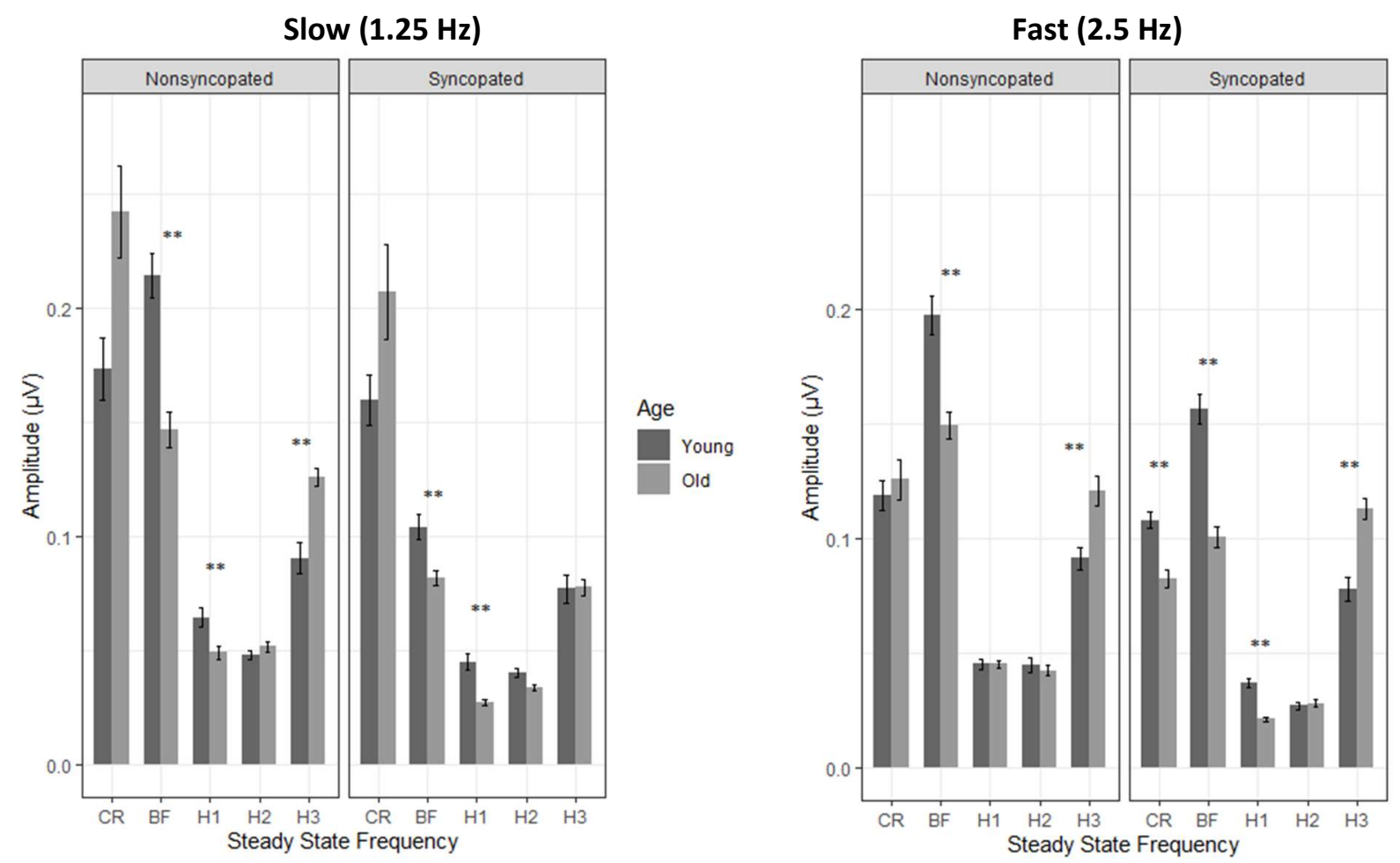

Figure 3. Mean amplitude of neural activity at the beat frequency, first three harmonics and cycle rate (meter frequency) for slow and fast tempi. Error bars indicate standard error of the mean, $* *=p<$ .003 . 

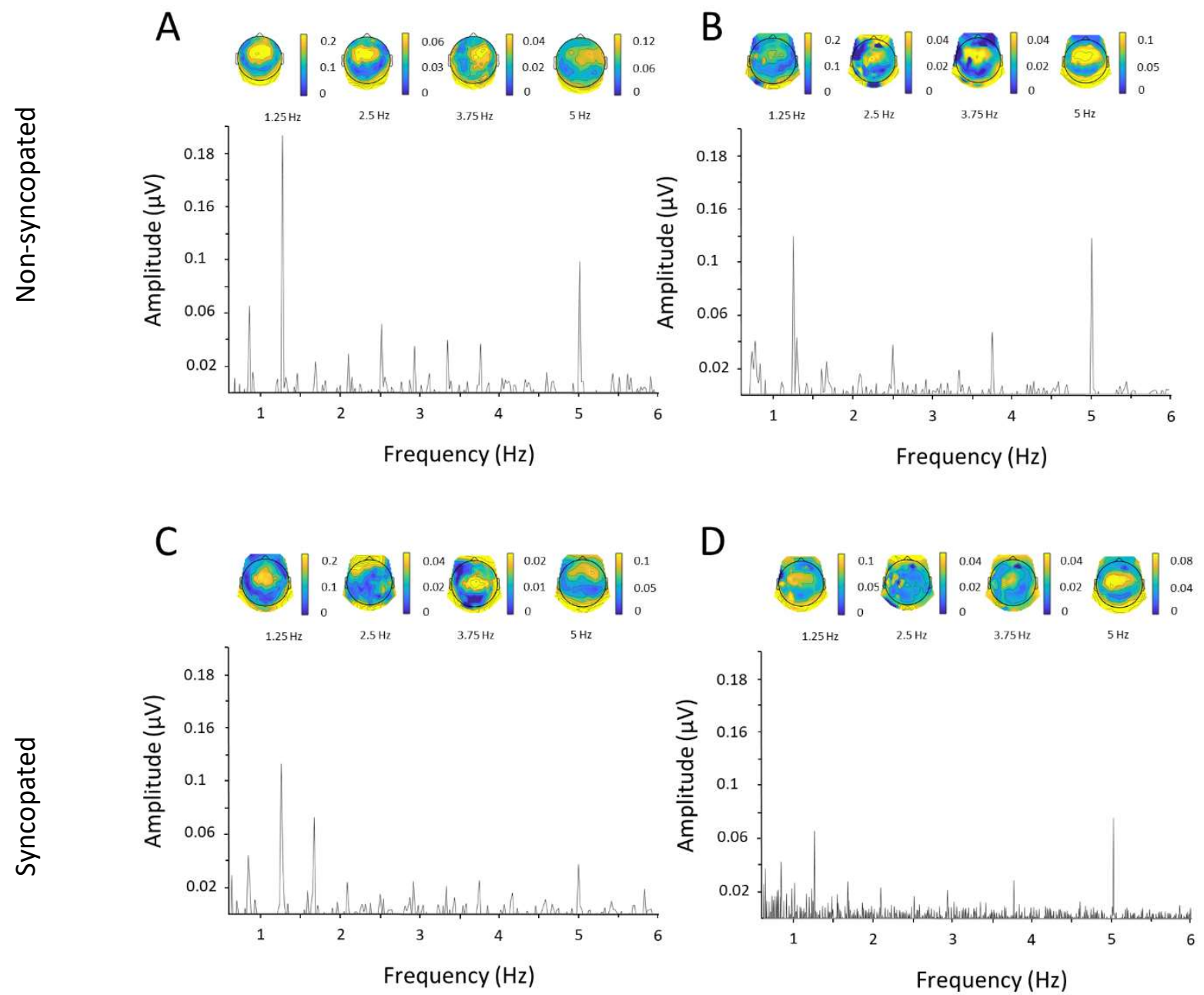

Figure 4. Amplitude of neural activity at electrode $\mathrm{Cz}$ ranging from $0.5 \mathrm{~Hz}$ to $6 \mathrm{~Hz}$ for young $(\mathrm{A}, \mathrm{C})$ and old (B, D) adults for non-syncopated (A, B) and syncopated (C, D) rhythms for slow tempo, along with topographic maps corresponding to each extracted beat frequency and the first three harmonics. Frequency and Amplitude scales are identical in all conditions to allow the relative comparison of amplitude peaks while topographical scales vary to all the relative comparison of activation distribution patterns across conditions. 

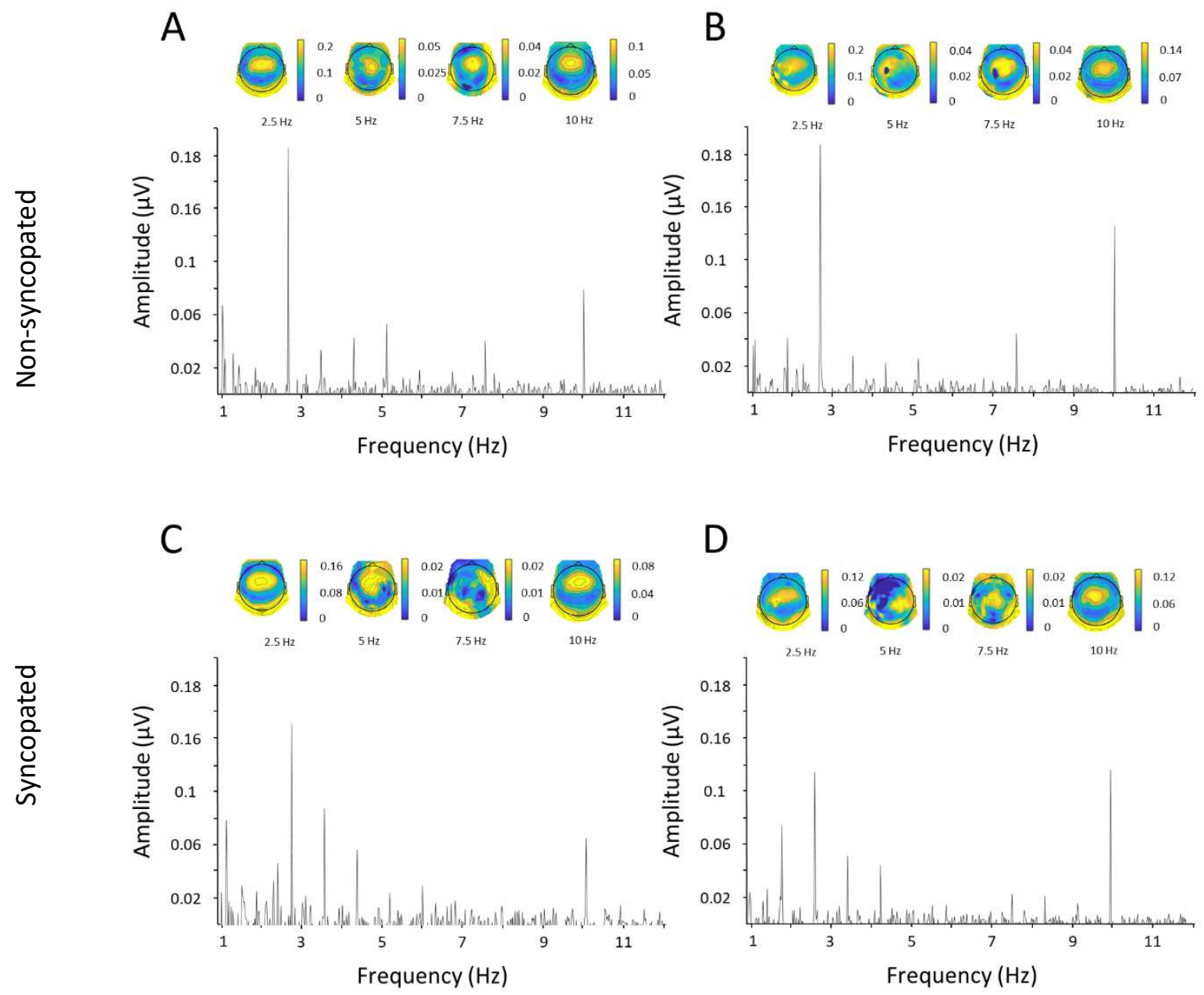

$\mathrm{D}$
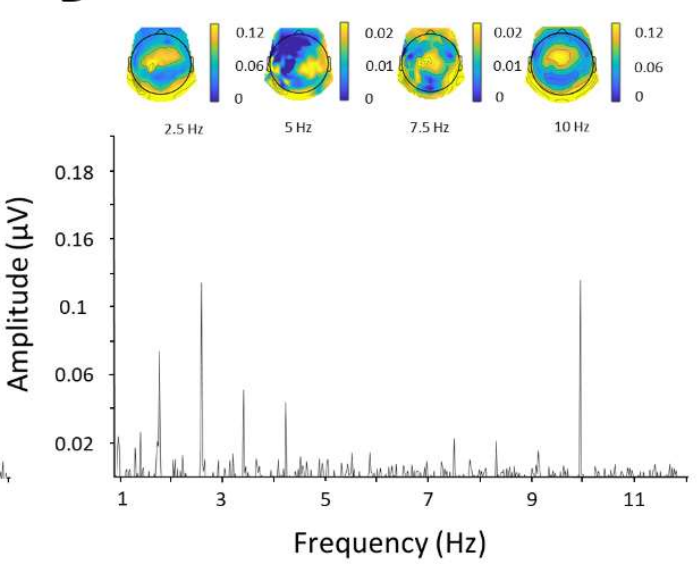

Figure 5 Amplitude of neural activity at electrode $\mathrm{Cz}$ ranging from $0.9 \mathrm{~Hz}$ to $12 \mathrm{~Hz}$ for young $(\mathrm{A}, \mathrm{C})$ and old (B, D) adults for non-syncopated (A, B) and syncopated (C, D) rhythms for fast tempo, along with topographic maps corresponding to each extracted beat frequency and the first three harmonics. Frequency and Amplitude scales are identical in all conditions to allow the relative comparison of amplitude peaks while topographical scales vary to all the relative comparison of activation distribution patterns across conditions. 


\section{Discussion}

In this study we examined differences between older and younger adults in neural entrainment to slow and fast syncopated and non-syncopated rhythms. In both younger and older adults, we observed neural activity at the beat frequency in all conditions with larger amplitude for slow vs. fast tempi and for non-syncopated vs. syncopated rhythms. In all cases, the amplitude was larger at meterrelated frequencies compared to meter-unrelated frequencies in both groups, irrespective of the prominence of meter frequencies in the stimuli. This replicates the findings reported in previous work and extends it to older population (Lenc et al., 2018; Nozaradan, Keller, Rossion, \& Mouraux, 2018; Nozaradan et al., 2016).

In older adults we observe lower amplitude at the BF across all stimulus conditions compared to younger adults (Figure 3). Furthermore, in older adults the amplitude at the CR was larger than the BF at slow tempi and also larger at the BF's third harmonic than in younger adults overall and not differentiated from the BF. In other words, while younger adults demonstrate the largest amplitude at BF (except in the slow syncopated condition) and H3 at approximately half the amplitude (Figure 3), older adults do not show such consistency or differentiation. This could be consistent with the compensation hypothesis of aging, where older adults increase activity at the $\mathrm{CR}$ and the $\mathrm{H} 3$ in order to compensate for the decrease in activity at the BF. Crucially, similarly to Sauvé et al. (2019), the difference in pure tone audiometric thresholds between age groups did not have a significant effect on neural activity amplitudes and so differences observed between age groups cannot here be attributed to low-level hearing abilities.

The age effect observed at the BF, where older adults have lower amplitude than younger adults, is in line with the findings of Sauvé et al. (2019), where participants were listening to an isochronous rhythm. Sauvé et al. (2019) also observed a lack of selectivity for the stimulus beat frequency in older adults as compared to younger adults, a finding we also observe here where the BF 
and $\mathrm{H} 3$ are poorly differentiated. This suggests that the active processing of complex rhythmic patterns, similarly to the automatic processing of auditory temporal regularities, is reduced and less specific in older adults, regardless of tempo and level of syncopation. This is consistent with an inhibition theory of aging (Salthouse \& Meinz, 1995), where the automatic inhibition of non-beat information is poorer in older adults. Here, this corresponds to supra- and sub-divisions of the beat.

The amplitude of the neural activity at the CR was larger in older adults compared to younger adults at slow tempi. This suggests that older adults attend to larger metrical groupings more than younger adults. The absence of this difference at the fast tempo and the inversion of this pattern for the syncopated rhythm suggests increased difficulty in isolating the beat frequency for older adults, where younger adults do so very clearly. This suggests that extracting the beat and forming higher order groupings is more difficult for older adults only when the stimulus rate is fast. This provides further support for the idea that older adults have more difficulty processing rapidly presented information (Andrews, Dowling, Bartlett, \& Halpern, 1998; Dowling, Bartlett, Halpern, \& Andrews, 2008; Salthouse, 1996). Alternatively, it is possible that the fixed order of our stimulus led to fatigue and therefore poorer performance. We consider this explanation unlikely as the study took less than thirty minutes to complete and participants were visibly engaged. Our data extends existing behavioural knowledge on age-related decline in speeded processing by providing neurophysiological evidence of such a decline in the context of neural entrainment to a beat.

The amplitude of the neural activity at $\mathrm{H} 3$ was larger in older adults compared to younger adults across all conditions except the slow syncopated condition. Its importance relative to $\mathrm{H} 1$ and $\mathrm{H} 2$ in both age groups may be a reflection of enculturation, where the vast majority of Western tonal music is divided into groups of four (Huron, 2006), leading the brain to produce activity at both the beat frequency and the frequency of the four-way subdivision more strongly than other subdivision groupings. If this is true, older adults' larger amplitude at H3 may be a culturally-based compensatory 
mechanism that makes up for the weaker encoding of the BF. This is congruent with our behavioural tapping data, where older adults tended to tap earlier than the beat than younger adults but with similar precision. However, a larger quantity and better quality of tapping data is needed to confirm a relationship between neural activity amplitude and tapping performance. It could also be a preference for responding at the note-level, as suggested when similar larger amplitude at the $\mathrm{H} 3$ was observed in Nozaradan et al. (Nozaradan, Schönwiesner, Keller, Lenc, \& Lehmann, 2018). Another alternative explanation for this is that the frequency of $\mathrm{H} 3$ is more prominent in the stimuli itself compared to $\mathrm{H} 1$ and $\mathrm{H} 2$; however, this cannot explain the age-related differences observed in the current study. In the current study, the higher amplitude at $\mathrm{H} 3$ in older adults was tempo-dependent, where the increase was more pronounced at the fast tempo. As such an increase in amplitude at H3 was not observed in Sauvé et al. (2019) and older adults here show a preference for the high-level measure, it is possible that this higher amplitude at the four-way subdivision observed in both age groups is a direct result of active engagement with the rhythms as opposed to passive exposure, or as a result of complex rhythms as opposed to an isochronous metronome. Future work will be necessary to identify the most accurate interpretation of the apparent enhancement of amplitude at $\mathrm{H} 3$.

Our data are consistent with both the compensation and inhibition theory of aging. Larger amplitudes at the $\mathrm{CR}$ and $\mathrm{H} 3$ suggest the recruitment of additional, musically relevant information to perform the given beat perception task. However, these could also indicate poor inhibition of these beat-related cues, leading to a lack of differentiation between these and the target beat. While our data cannot identify the best interpretation, similar lack of differentiation in older adults has been observed in frequency tagging for a visual feature-selection task (Quigley, Andersen, Schulze, Grunwald, \& Müller, 2010), where older and younger adults attended to movement of differently coloured dots on a screen. Steady-state visual evoked potential in response to each colour's motion was compared: younger adults showed clear differentiation between the relevant and irrelevant stimulus while older adults did not. 
Behaviourally, older adults successfully detected motion overall but also discriminated poorly between relevant and irrelevant stimuli. This behavioural data points more strongly to the inhibition theory of aging as the mechanism at play. However, no such age effects were observed for spatial attention tasks (Govenlock, Sekuler, \& Bennett, 2010; Quigley, Andersen, \& Müller, 2012), suggesting that such age effects are task-specific.

To summarize, this study has demonstrated differences between older and younger adults on neural entrainment to a rhythmic stimulus. In older adults, we observed increased amplitude at the measure frequency at slow tempi, a lack of differentiation between meter-related frequencies at fast tempi and an increased amplitude at the beat frequency's third harmonic (also coinciding with the frequency of the smallest intervals in the rhythms), which may be a culturally-based compensatory mechanism used to offset the lower amplitude at the beat frequency itself. Here we found that older adults had a larger amplitude of $\mathrm{H} 3$ as compared to $\mathrm{H} 1$ and $\mathrm{H} 2$, along with an increased reliance on higher level groupings. This finding is consistent with existing evidence that aging does not seem to affect performance on music tasks (Halpern et al., 1995, 1998, 1996, 2017) but that in some cases neural compensatory mechanisms are deployed (Halpern et al., 2017). 


\section{References}

Andrews, M. W., Dowling, W. J., Bartlett, J. C., \& Halpern, A. R. (1998). Identification of speeded and slowed familiar melodies by younger, middle-aged, and older musicians and nonmusicians. Psychology and Aging, 13(3), 462.

Barr, D. J., Levy, R., Scheepers, C., \& Tily, H. J. (2013). Random effects structure for confirmatory hypothesis testing: Keep it maximal. Journal of Memory and Language, 68(3), 255-278. https://doi.org/10.1016/j.jml.2012.11.001

Bates, D., Mächler, M., Bolker, B., \& Walker, S. (2015). Fitting Linear Mixed-Effects Models Using Ime4. Journal of Statistical Software, 67(1). https://doi.org/10.18637/jss.v067.i01

Birren, J. E., \& Fisher, L. M. (1995). Aging and speed of behavior: Possible consequences for psychological functioning. Annual Review of Psychology, 46(1), 329-353.

Cabeza, R. (2002). Hemispheric asymmetry reduction in older adults: The HAROLD model. Psychology and Aging, 17(1), 85 .

Cabeza, R., Daselaar, S. M., Dolcos, F., Prince, S. E., Budde, M., \& Nyberg, L. (2004). Task-independent and task-specific age effects on brain activity during working memory, visual attention and episodic retrieval. Cerebral Cortex, 14(4), 364-375.

Craik, F. I., \& Salthouse, T. A. (2011). The handbook of aging and cognition. Psychology press.

Davis, S. W., Kragel, J. E., Madden, D. J., \& Cabeza, R. (2011). The architecture of cross-hemispheric communication in the aging brain: Linking behavior to functional and structural connectivity. Cerebral Cortex, 22(1), 232-242.

Dowling, W. J., Bartlett, J. C., Halpern, A. R., \& Andrews, M. W. (2008). Melody recognition at fast and slow tempos: Effects of age, experience, and familiarity. Attention, Perception, \& Psychophysics, 70(3), 496-502. 
Finkel, D., Reynolds, C. A., McArdle, J. J., \& Pedersen, N. L. (2007). Age changes in processing speed as a leading indicator of cognitive aging. Psychology and Aging, 22(3), 558.

Gazzaley, A., Cooney, J. W., Rissman, J., \& D’esposito, M. (2005). Top-down suppression deficit underlies working memory impairment in normal aging. Nature Neuroscience, 8(10), 1298.

Govenlock, S. W., Sekuler, A. B., \& Bennett, P. J. (2010). Assessing the effect of aging on spatial frequency selectivity selectivity of visual mechanisms with the steady state visually evoked potential (ssVEP). Journal of Vision, 10(7), 481-481.

Grady, C. L., McIntosh, A. R., \& Craik, F. I. (2003). Age-related differences in the functional connectivity of the hippocampus during memory encoding. Hippocampus, 13(5), 572-586.

Halpern, A. R., Bartlett, J. C., \& Dowling, W. J. (1995). Aging and experience in the recognition of musical transpositions. Psychology and Aging, 10(3), 325.

Halpern, A. R., Bartlett, J. C., \& Dowling, W. J. (1998). Perception of mode, rhythm, and contour in unfamiliar melodies: Effects of age and experience. Music Perception: An Interdisciplinary Journal, 15(4), 335-355.

Halpern, A. R., Kwak, S., Bartlett, J. C., \& Dowling, W. J. (1996). Effects of aging and musical experience on the representation of tonal hierarchies. Psychology and Aging, 11(2), 235.

Halpern, A. R., Zioga, I., Shankleman, M., Lindsen, J., Pearce, M. T., \& Bhattarcharya, J. (2017). That note sounds wrong! Age-related effects in processing of musical expectation. Brain and Cognition, $113,1-9$.

Hasher, L. (2015). Inhibitory Deficit Hypothesis. In The Encyclopedia of Adulthood and Aging (pp. 1-5). https://doi.org/10.1002/9781118521373.wbeaa259

Hasher, L., \& Zacks, R. T. (1988). Working memory, comprehension, and aging: A review and a new view. In Psychology of learning and motivation (Vol. 22, pp. 193-225). Elsevier. 
Horn, J. L. (1982). The theory of fluid and crystallized intelligence in relation to concepts of cognitive psychology and aging in adulthood. In Aging and cognitive processes (pp. 237-278). Springer.

Horn, J. L., \& Cattell, R. B. (1967). Age differences in fluid and crystallized intelligence. Acta Psychologica, $26,107-129$.

Huron, D. (2006). Sweet anticipation: Music and the psychology of expectation. Cambridge, MA, US: The MIT Press. (2006-08683-000).

Lagrois, M.-É., Peretz, I., \& Zendel, B. R. (2018). Neurophysiological and Behavioral Differences between Older and Younger Adults When Processing Violations of Tonal Structure in Music. Frontiers in Neuroscience, 12, 54.

Large, E. W. (2008). Resonating to musical rhythm: Theory and experiment. The Psychology of Time, 189-232.

Lenc, T., Keller, P. E., Varlet, M., \& Nozaradan, S. (2018). Neural tracking of the musical beat is enhanced by low-frequency sounds. Proceedings of the National Academy of Sciences, 115(32), 82218226.

Lustig, C., Hasher, L., \& Zacks, R. T. (2007). Inhibitory deficit theory: Recent developments in a "new view." Inhibition in Cognition, 17, 145-162.

Madden, D. J. (1992). Four to ten milliseconds per year: Age-related slowing of visual word identification. Journal of Gerontology, 47(2), P59-P68.

Mouraux, A., \& lannetti, G. D. (2008). Across-trial averaging of event-related EEG responses and beyond. Magnetic Resonance Imaging, 26(7), 1041-1054. https://doi.org/10.1016/j.mri.2008.01.011

Mouraux, André, lannetti, G. D., Colon, E., Nozaradan, S., Legrain, V., \& Plaghki, L. (2011). Nociceptive steady-state evoked potentials elicited by rapid periodic thermal stimulation of cutaneous nociceptors. Journal of Neuroscience, 31(16), 6079-6087. 
Nozaradan, S., Keller, P. E., Rossion, B., \& Mouraux, A. (2018). EEG frequency-tagging and input-output comparison in rhythm perception. Brain Topography, 31(2), 153-160.

Nozaradan, S., Peretz, I., \& Keller, P. E. (2016). Individual differences in rhythmic cortical entrainment correlate with predictive behavior in sensorimotor synchronization. Scientific Reports, 6, 20612.

Nozaradan, S., Peretz, I., Missal, M., \& Mouraux, A. (2011). Tagging the neuronal entrainment to beat and meter. Journal of Neuroscience, 31(28), 10234-10240.

Nozaradan, S., Peretz, I., \& Mouraux, A. (2012). Selective neuronal entrainment to the beat and meter embedded in a musical rhythm. Journal of Neuroscience, 32(49), 17572-17581.

Nozaradan, S., Schönwiesner, M., Keller, P. E., Lenc, T., \& Lehmann, A. (2018). Neural bases of rhythmic entrainment in humans: Critical transformation between cortical and lower-level representations of auditory rhythm. European Journal of Neuroscience, 47(4), 321-332.

Pichora-Fuller, M. K., Schneider, B. A., \& Daneman, M. (1995). How young and old adults listen to and remember speech in noise. The Journal of the Acoustical Society of America, 97(1), 593-608.

Quigley, C., Andersen, S. K., \& Müller, M. M. (2012). Keeping focused: Sustained spatial selective visual attention is maintained in healthy old age. Brain Research, 1469, 24-34.

Quigley, C., Andersen, S. K., Schulze, L., Grunwald, M., \& Müller, M. M. (2010). Feature-selective attention: Evidence for a decline in old age. Neuroscience Letters, 474(1), 5-8.

Reuter-Lorenz, P. A. (2002). New visions of the aging mind and brain. Trends in Cognitive Sciences, 6(9), 394-400.

Reuter-Lorenz, P. A., \& Cappell, K. A. (2008). Neurocognitive aging and the compensation hypothesis. Current Directions in Psychological Science, 17(3), 177-182.

Reuter-Lorenz, P. A., Jonides, J., Smith, E. E., Hartley, A., Miller, A., Marshuetz, C., \& Koeppe, R. A. (2000). Age differences in the frontal lateralization of verbal and spatial working memory revealed by PET. Journal of Cognitive Neuroscience, 12(1), 174-187. 
Salthouse, T. A. (1990). Working memory as a processing resource in cognitive aging. Developmental Review, 10(1), 101-124.

Salthouse, T. A. (1994). The aging of working memory. Neuropsychology, 8(4), 535.

Salthouse, T. A. (1996). The processing-speed theory of adult age differences in cognition. Psychological Review, 103(3), 403.

Salthouse, T. A., \& Babcock, R. L. (1991). Decomposing adult age differences in working memory. Developmental Psychology, 27(5), 763.

Salthouse, T. A., \& Meinz, E. J. (1995). Aging, inhibition, working memory, and speed. The Journals of Gerontology Series B: Psychological Sciences and Social Sciences, 50(6), P297-P306.

Sauvé, S. A., Bolt, E. L., Fleming, D., \& Zendel, B. R. (2019). The impact of aging on neurophysiological entrainment to a metronome. NeuroReport.

Sauvé, S. A., Sayed, A., Dean, R. T., \& Pearce, M. T. (2018). Effects of pitch and timing expectancy on musical emotion. Psychomusicology: Music, Mind, and Brain, 28(1), 17-39. http://dx.doi.org/10.1037/pmu0000203 